\title{
Varfarina: uma revisão baseada na evidência das interacções alimentares e medicamentosas
}

Nuno Lima*

\section{RESUMO}

Introdução: A varfarina tem sido o pilar da terapêutica anticoagulante oral. A sua eficácia já foi comprovada em numerosos contextos na prática clínica.

Objectivos: Rever quais são as interacções alimentares e medicamentosas da varfarina.

Metodologia: Foi realizada uma pesquisa nas bases de dados British Medical Journal (BMJ) Clinical Evidence, Evidence Based Medicine online, National Guideline Clearinghouse, National Library of Guidelines, The Cochrane Library, DARE, Bandolier e MED$L I N E$, utilizando as seguintes palavras-chave (termos MESH): warfarin, drug interactions, food interactions e herbal interactions. A pesquisa foi limitada a artigos escritos em inglês, francês, espanhol ou português e publicados desde Janeiro de 2003 até ao presente.

Foi também efectuada uma pesquisa no Índex de Revistas Médicas Portuguesas, com as seguintes palavras-chave: varfarina, interacções alimentares e interacções medicamentosas.

Para avaliar o nível de evidência foi utilizada a escala da American Family Physician (Strenght Of Recommendation TaxonomySORT): nível A (ensaios clínicos aleatorizados controlados/meta-análises); nível B (outra evidência) e nível C (consensos/opinião de peritos).

Resultados e discussão: Interacções alimentares: Os alimentos ricos em vitamina K estão envolvidos na interacção com a varfarina. Não está indicada a sua evicção absoluta mas recomenda-se uma ingestão moderada e sem grandes flutuações.

Interaç̧ões medicamentosas: São numerosas e ocorrem por diversos mecanismos: (1) Diminuição da absorção da varfarina; (2) Potenciação/Inibição do citocromo P450 a nível hepático; (3) Diminuição da síntese de vitamina K endógena pela flora intestinal; (4) Inibição da agregação plaquetária; (5) Aumento do catabolismo dos factores de coagulação; (6) Indução dos factores da coagulação; (7) Mecanismos desconhecidos.

Interacções com produtos naturais: a erva de São João (chá de hipericão), em indivíduos saudáveis, aumenta a metabolização da varfarina e inibe, desta forma, o seu efeito anticoagulante.

Conclusão: São escassos os estudos com bom nível de evidência, sobre um tema tão importante como este. São necessários mais ensaios clínicos aleatorizados e controlados, de longa duração e de boa qualidade, sobre as interacções alimentares e medicamentosas da varfarina.

Palavras-chave: Varfarina; Interacções Alimentares; Interacções Medicamentosas.

\section{INTRODUÇÃO}

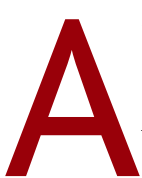
varfarina tem sido o pilar da terapêutica anticoagulante oral desde há cerca de 50 anos. A sua eficácia já foi comprovada na prevenção do tromboembolismo venoso, na prevenção da embolia sistémica em doentes com próteses valvulares cardíacas ou fibrilação auricular e na prevenção primária do enfarte agudo do miocárdio (EAM) em ho-

*Médico Interno de Medicina Geral e Familiar.

Centro de Saúde de Paranhos. mens de alto risco. Revelou ainda utilidade, em doentes que já tiveram um EAM, na prevenção da recorrência de enfarte e da ocorrência de Acidente Vascular Cerebral (AVC) ou morte. ${ }^{1}$

A varfarina inibe maioritariamente a enzima epoxireductase da vitamina $\mathrm{K}$, o que impede a interconversão cíclica desta vitamina. Desta forma, ao bloquear a interconversão cíclica da vitamina $\mathrm{K}$, impede a carboxilação dos resíduos de glutamato nas regiões terminais das proteínas dependentes da vitamina $\mathrm{K}$ - factores II, 
VII, IX e X e proteínas C e S (Figura 1). A carboxilação é necessária a uma alteração conformacional (dependente do cálcio) que ocorre nas proteínas da coagulação atrás descritas e que leva à ligação a cofactores nas superfícies fosfolipídicas nos locais de lesão vascular. ${ }^{1}$ Desta forma, na presença da varfarina, os factores da coagulação dependentes da vitamina $\mathrm{K}$ perdem a sua actividade e não se conseguem ligar aos locais de lesão vascular, dificultando o controlo da hemorragia.

No entanto, a vitamina K exógena (proveniente da dieta, por exemplo) pode contornar o antagonismo da varfarina, conseguindo produzir o cofactor (vitamina KH2) necessário à carboxilação das proteínas da coagulação dependentes da vitamina $K$, através duma via menos sensível à varfarina (a da enzima vitamina $\mathrm{K}$ reductase). ${ }^{2}$

A varfarina é o anticoagulante oral mais utilizado na prática clínica e é composta por dois isómeros, a R-varfarina e a S-varfarina (5 vezes mais potente que o isómero R), que são metabolizados no fígado por vias diferentes. ${ }^{1}$

As primeiras proteínas da coagulação dependentes da vitamina K a serem afectadas (após o início da toma

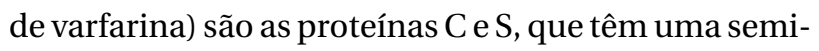
-vida muito curta. Desta forma, os seus níveis diminuem rapidamente, o que origina uma fase pró-coagulante no início da terapêutica com varfarina. ${ }^{1,3}$ De seguida diminui o factor VII (semi-vida de, aproximadamente, 6 horas) e depois o factor X e IX. O efeito antitrombótico da varfarina apenas é obtido quando diminuem os níveis do factor II, o qual tem uma semi-vida de cerca de 60 a 72 horas. Isto implica que, durante os primeiros 4 dias de terapêutica com varfarina, se utilize também a heparina, que tem um efeito imediato. ${ }^{1}$

Com o avanço da tecnologia médica, a esperança média de vida tem vindo a aumentar progressivamente. Cerca de 2 milhões de pessoas atingiram os 65 anos em 2002 e espera-se que, em 2030, este número chegue aos 70 milhões e represente $20 \%$ da população. Aliado a este facto, cerca de $79 \%$ da população com idade maior ou igual a 65 anos apresenta, pelo menos, um critério clínico que justifica a toma de varfarina. No entanto, apenas cerca de 15 a $44 \%$ destes doentes, sem contraindicações para a anticoagulação oral com varfarina, faz este tratamento. ${ }^{4}$

A terapêutica com varfarina é monitorizada pela me-

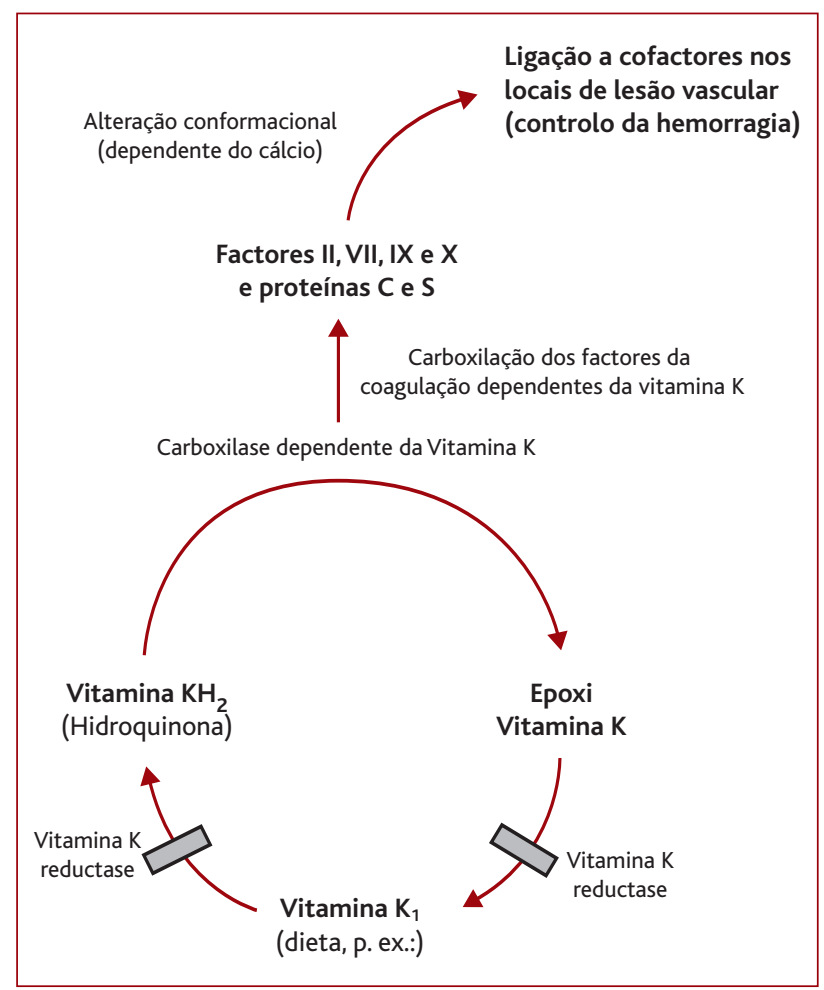

Figura 1. Ciclo da vitamina K. A varfarina inibe a acção das reductases (barras), inibindo a síntese de vitamina $\mathrm{K}_{1}$ e vitamina $\mathrm{KH}_{2}$. Desta forma impede a carboxilação (activação) dos factores da coagulação dependentes da vitamina $K$, o que resulta num controlo menos eficaz da hemorragia.

dição do INR (International Normalized Ratio) que é uma medida normalizada a nível internacional para minimizar as variações nos valores do tempo de protrombina (PT) entre laboratórios diferentes. ${ }^{1,3,5-7}$

A varfarina é um fármaco com uma janela terapêutica estreita e que exibe uma enorme variabilidade em termos de dose-resposta de pessoa para pessoa. Além disso, é alvo de numerosas interacções alimentares e medicamentosas, que podem ser responsáveis pelo aumento do seu efeito anticoagulante (que pode cursar com hemorragias graves e/ou fatais) ou pela inibição da sua acção, colocando o doente em risco de sofrer um evento trombótico. ${ }^{1}$

Este último ponto coloca um desafio enorme ao médico de família, uma vez que muitos dos doentes que necessitam de ser tratados com varfarina são de idade avançada e estão polimedicados, tornando-se essencial ter conhecimento dos fármacos que interagem com ela. 
Ainda no campo das interacções medicamentosas não podemos esquecer os medicamentos naturais de venda livre e os chás de ervas que os doentes não mencionam que tomam, por acharem inofensivos, uma vez que são substâncias naturais.

Outra dificuldade que a terapêutica com varfarina coloca é a alimentação do doente, que se trata de um aspecto de enorme variabilidade interindividual e ao longo do tempo no mesmo doente, principalmente no que toca a alimentos ricos em vitamina $\mathrm{K} .^{5}$

Sendo assim, uma vez que a utilização deste fármaco tende a aumentar e tendo em conta a complexidade do início e da monitorização desta terapêutica, percebe-se a importância da realização duma revisão baseada na evidência acerca das interacções alimentares e medicamentosas da varfarina.

Neste contexto, foi efectuada uma revisão baseada na evidência, com o objectivo de rever quais são as interacções alimentares e medicamentosas da varfarina.

\section{MÉTODOS}

Foi realizada uma pesquisa nas bases de dados British Medical Journal (BMJ) Clinical Evidence, Evidence Based Medicine online, National Guideline Clearinghouse, National Library of Guidelines, The Cochrane Library, DARE, Bandolier e MEDLINE, de sistemas, sinopses, guidelines, meta-análises, revisões sistemáticas e ensaios clínicos aleatorizados e controlados, publicados desde Janeiro de 2003 até ao presente. Foram admitidos na pesquisa os artigos que estivessem escritos em inglês, francês, espanhol e português e foram utilizadas as seguintes palavras-chave (termos $\mathrm{MeSH}$ ): warfarin, drug interactions, food interactions e herbal interactions.

No Índex de Revistas Médicas Portuguesas, a pesquisa foi feita com os seguintes termos: varfarina, interacções medicamentosas e interacções alimentares.

Os critérios utilizados para a inclusão dos artigos nesta revisão foram os seguintes:

- População: indivíduos medicados com varfarina, independentemente da situação clínica que motiva a sua utilização e da idade e/ou sexo dos doentes.

- Intervenção: administração diária de varfarina, durante um período mínimo de quatro semanas.

- Comparação: varfarina versus alimentos ricos em vitamina $K$, varfarina versus outros fármacos e varfari- na versus medicamentos naturais e/ou chás de ervas. - Resultados: potenciação do efeito anticoagulante da varfarina, que resulte em hemorragia (de menor ou maior gravidade) ou inibição do seu efeito levando à ocorrência dum evento trombótico ou embólico (ou colocando o doente numa posição de risco elevado para um destes desfechos).

A pesquisa efectuada resultou na identificação de 104 artigos. Destes, foram excluídos os artigos repetidos, aqueles em que se verificou discordância com o objectivo da revisão e aqueles que não cumpriam os critérios de inclusão previamente descritos. Obtiveram-se, deste modo, 9 artigos (duas guidelines, três revisões sistemáticas e quatro ensaios clínicos aleatorizados controlados), que foram incluídos na revisão. Surpreendentemente, não se encontrou nenhum artigo de revisão baseada na evidência, sobre um tema com grande relevância para a prática clínica de profissionais de saúde das mais variadas áreas.

Foram ainda seleccionados alguns artigos (contidos nas referências dos anteriores ou através da pesquisa por artigos relacionados), considerados relevantes para esta revisão, obtendo-se assim um total de 21 artigos.

Para avaliar o nível de evidência, foi utilizada a escala da American Family Physician (Strenght Of Recommendation Taxonomy-SORT): nível A (ensaios clínicos aleatorizados controlados/meta-análises); nível B (outra evidência) e nível C (consensos/opinião de peritos). ${ }^{8}$

\section{CORPO DE REVISÃO}

Para uma maior compreensão os resultados foram agrupados em interacções alimentares, interacções medicamentosas e interacções com produtos naturais.

\section{Interacções alimentares}

Da leitura dos artigos ficou bem claro que os alimentos ricos em vitamina Kinteragem com a varfarina, uma vez que a vitamina K exógena pode contornar o antagonismo da varfarina, conseguindo produzir o cofactor (vitamina $\mathrm{KH}_{2}$ ) necessário à carboxilação das proteínas da coagulação dependentes da vitamina $\mathrm{K}$ (factores II, VII, IX e X e proteínas C e S), através duma via menos sensível à varfarina (a da enzima vitamina $\mathrm{K}$ reductase). . $, 2,5-7,9-11^{-11}$

Da análise da porção de vitamina K presente em vários alimentos ${ }^{2}$ concluiu-se que esta está presente em 
maior quantidade nos alimentos de origem vegetal, especialmente nos de cor verde-escura e com folhas. Tal é o caso dos espinafres, couve-galega, couve-tronchuda, couve-de-bruxelas, alfafa, espargo, abacate, alface, grelos, salsa e bróculos. Geralmente, quanto mais verde for a planta maior a sua quantidade de vitamina $\mathrm{K}$. $\mathrm{O}$ facto de congelar, cozer ou aquecer estes vegetais no micro-ondas não altera significativamente o seu conteúdo em vitamina $\mathrm{K}^{2,7}$

Outras fontes de vitamina K da dieta são a couve-flor, as sementes de uva, o leite de soja, o azeite e os óleos de soja, de canola e de semente de algodão. Os temperos de saladas, as margarinas, a maionese, os produtos de pastelaria e outros alimentos, preparados com estes óleos, também podem ser fontes de vitamina $\mathrm{K}$ a ter em conta. ${ }^{2}$

Por outro lado, os óleos de amendoim, de girassol, de açafrão e de sésamo têm um conteúdo limitado de vitamina K. ${ }^{2}$

Sendo assim, no que diz respeito às interacções alimentares, a evidência confirmou que os alimentos ricos em vitamina K estão envolvidos na interacção com a varfarina (nível de evidência A). ${ }^{12}$

Não está indicado que os doentes a tomar varfarina evitem, em absoluto, os alimentos ricos em vitamina $\mathrm{K}$, acima referidos. Recomenda-se, no entanto, que façam uma ingestão moderada e sem grandes flutuações dos mesmos (nível de evidência B). ${ }^{2,5-7}$

\section{Interacções medicamentosas}

As interacções medicamentosas com a varfarina podem ocorrer através de diversos mecanismos.

\section{Diminuição da absorção da varfarina}

Uma das formas de interacção com a varfarina ocorre pela diminuição da sua absorção (e consequente diminuição do seu efeito) obtida pelas resinas sequestradoras de ácidos biliares (colestiramina e colestipol) utilizadas no tratamento das dislipidemias. Sendo assim, a varfarina não deve ser tomada nas 2 horas anteriores nem nas 6 horas seguintes à toma destas resinas (nível de evidência B). .,10,11 $^{-1}$

Potenciação/Inibição do citocromo $P_{450}$ a nível hepático Outra forma de interacção com a varfarina ocorre pela potenciação ou inibição do citocromo $\mathrm{P}_{450}$ a nível hepático. Tal como foi dito antes, a varfarina é composta por uma mistura racémica de dois isómeros: a R-varfarina e a S-varfarina (5 vezes mais potente que a anterior). A S-varfarina é metabolizada pelo citocromo $\mathrm{P}_{450}$ CYP2C9 enquanto que a R-varfarina é metabolizada pelos citocromos $\mathrm{P}_{450}$ CYP1A2 e CYP3A4. Por este motivo, os fármacos capazes de interferir com o citocromo CYP2C9 são responsáveis por interacções significativas com a varfarina, enquanto os fármacos que interferem com os citocromos CYP1A2 e CYP3A4 não provocam interacções clinicamente significativas (Quadro I). ${ }^{5,10}$

Em suma, outra forma de interacção com a varfarina ocorre pela potenciação ou inibição do citocromo $\mathrm{P}_{450}$ CYP2C9 a nível hepático, mecanismo responsável pela interacção com diversos fármacos - Quadro I (nível de evidência B). ${ }^{1,5-7,9-11,13,14}$

\section{Diminuição da síntese de vitamina K pela flora intestinal}

Outro mecanismo de acção responsável por interacções medicamentosas com a varfarina passa pela redução da síntese de vitamina $\mathrm{K}$ pela flora intestinal (da qual depende, em parte, o pool de vitamina K endógeno). Isto explica que alguns antibióticos que alteram a flora intestinal e que diminuem, deste modo, a quantidade de vitamina K disponível, potenciem o efeito da varfarina. Por conseguinte, os profissionais de saúde devem ter cautela ao introduzirem um antibiótico (independentemente de qual seja) num doente medicado com varfarina, principalmente quando se trata dum antibiótico que também inibe a sua metabolização pelo citocromo $\mathrm{P}_{450}$ CYP2C9 (sulfametoxazol, metronidazol e, em menor escala, os macrólidos e as quinolonas)., ${ }^{1,10}$

Estas afirmações apresentam um nível de evidência B. ${ }^{1,10}$

\section{Inibição da agregação plaquetária}

Os fármacos que inibem a agregação plaquetária também são responsáveis por interacções medicamentosas com a varfarina. Este mecanismo não se trata duma interacção farmacocinética (como os anteriores) mas sim duma interacção farmacodinâmica. Como exemplos de fármacos que inibem a agregação plaquetária e que, por conseguinte, aumentam o risco de hemorragia através do efeito aditivo da varfarina, temos o ácido acetilsalicílico, o acetilsalicilato de lisina, o clopidogrel, a ticlopidina, o dipiridamol e os antiinflamatórios 


\begin{tabular}{|c|c|}
\hline \multicolumn{2}{|c|}{$\begin{array}{l}\text { QUADRO I. Interacções medicamentosas clinicamente } \\
\text { significativas com a varfarina }{ }^{1,5-7,9-11,13-18}\end{array}$} \\
\hline$(\downarrow$ INR) & ( $\uparrow$ INR) \\
\hline Estrogénios & $\begin{array}{l}\text { Acetilsalicilato de lisina } \\
\text { Ácido acetilsalicílico } \\
\text { AINEs } \\
\text { Clopidogrel } \\
\text { Dipiridamol } \\
\text { Inibidores selectivos da } \\
\text { recaptação da serotonina } \\
\text { Ticlopidina } \\
\text { Tiroxina } \\
\text { Paracetamol ( } \geq 4 \mathrm{~g} / \text { dia) }\end{array}$ \\
\hline $\begin{array}{l}\text { Aumento da metabolização } \\
\text { da varfarina ( } \downarrow \text { INR) }\end{array}$ & $\begin{array}{l}\text { Diminuição da } \\
\text { metabolização da } \\
\text { varfarina ( } \uparrow \text { INR) } \\
\end{array}$ \\
\hline Azatioprina & Amiodarona \\
\hline $\begin{array}{l}\text { Barbitúricos } \\
\text { (fenobarbital, tiopental) }\end{array}$ & Celecoxib \\
\hline Carbamazepina & Dissulfiram \\
\hline Ciclosporina & Eritromicina \\
\hline Clorodiazepóxido & Fluvoxamina \\
\hline Dicloxacilina & $\begin{array}{l}\text { Imidazóis (fluconazol, } \\
\text { voriconazol, miconazol, } \\
\text { itraconazol) }\end{array}$ \\
\hline $\begin{array}{l}\text { Fenitoína (inibição inicial da } \\
\text { metabolização da varfarina } \\
\text { e potenciação posterior) }\end{array}$ & Isoniazida \\
\hline Griseofulvina & Levofloxacina \\
\hline Rifampicina & $\begin{array}{l}\text { Metronidazol } \\
\text { Piroxicam } \\
\text { Propafenona } \\
\text { Sulfametoxazol } \\
\text { Tamoxifeno }\end{array}$ \\
\hline
\end{tabular}

não esteróides (AINEs). Além disso, o acetilsalicilato de lisina, o ácido acetilsalicílico e os AINEs, também podem provocar erosões gástricas, aumentando, desta forma, o risco de hemorragia digestiva alta. ${ }^{1,5,6,10,11}$ Foi ainda encontrado um artigo que fez referência aos inibidores selectivos da recaptação da serotonina (antidepressivos que são prescritos frequentemente em conjunto com a varfarina), que, ao diminuírem os níveis de serotonina das plaquetas, também podem inibir a agregação das mesmas. ${ }^{18}$
Estes achados têm um nível de evidência B. . $^{1,5,6,10,11,18}$

\section{Aumento do catabolismo dos factores da coagulação} Também se encontrou evidência de que a tiroxina potencia o efeito anticoagulante da varfarina (ampliando o risco hemorrágico) ao aumentar o catabolismo dos factores da coagulação (nível de evidência B).

\section{Indução dos factores da coagulação}

Os estrogénios (contraceptivos orais), através da indução dos factores da coagulação, têm um efeito pró-coagulante, contrariando, desta forma, o efeito anticoagulante da varfarina (nível de evidência B). ${ }^{7,11}$

\section{Mecanismos desconhecidos}

O consumo excessivo de álcool de forma aguda ou crónica, principalmente quando se associa a patologia hepática, resulta na inibição da metabolização da varfarina (e no aumento do INR) através dum mecanismo desconhecido. O consumo moderado de álcool não interage, significativamente, com a terapêutica com varfarina. No entanto, recomenda-se abstinência absoluta de álcool, uma vez que, mesmo quantidades moderadas de álcool podem aumentar o risco de quedas e/ou outros acidentes. ${ }^{10,11,14}$

Estes resultados apresentam um nível de evidência B. ${ }^{10,11,14}$

Três ensaios clínicos aleatorizados controlados mostraram que o paracetamol (numa dose igual ou superior a $4 \mathrm{~g} /$ dia) potencia o efeito anticoagulante da varfarina, por um mecanismo ainda não muito bem conhecido - nível de evidência A. ${ }^{15-17}$

Pensa-se que esta interacção aconteça por um efeito não-farmacocinético, uma vez que se observou um aumento do INR após a introdução de paracetamol ( $\geq 4 \mathrm{~g} /$ dia) mas a concentração de varfarina permaneceu constante. Verificou-se um aumento médio de 1,04 no INR, apenas 4 dias depois da toma concomitante de varfarina e de paracetamol (na dose previamente referida). ${ }^{15,16,17}$

Ainda assim, uma vez que não existe uma alternativa melhor ao paracetamol, quando um doente que toma varfarina necessita de um analgésico e/ou de um antipirético, o paracetamol deve ser o fármaco de primeira escolha (na menor dose e duração possíveis) - nível de evidência $C$. $^{15,16,17}$ 


\section{Interacções com produtos naturais}

O aumento da utilização de produtos naturais (chás, produtos de ervanária), muitas vezes desconhecido e não investigado pelo médico de família, coloca uma preocupação acrescida no que se refere à segurança e eficácia do tratamento com varfarina, um fármaco pródigo em interacções.

Estes produtos são de venda livre não havendo, por conseguinte, qualquer controlo sobre a sua aquisição e utilização. Outros produtos (ervas, por exemplo) são cultivados ou apanhados da terra pelo próprio doente que acredita num potencial de cura para as suas doenças. Deve ainda referir-se que estes produtos naturais ou os chás de ervas contêm uma mistura de substâncias activas e que a sua concentração depende da estação do ano em que foi feita a colheita, do estado de maturação e das condições em que cresceu a planta. Isto pode ter como consequência que o mesmo produto vegetal que foi adquirido em alturas diferentes venha a ter diferente actividade biológica.

Num estudo efectuado nos Estados Unidos estimou-se que, aproximadamente, $30 \%$ da população utiliza produtos naturais. ${ }^{19}$

Muitos doentes assumem que, uma vez que estes produtos são naturais, são seguros e podem ser tomados em conjunto com a medicação habitual. Por esta razão não sentem necessidade de informar os profissionais de saúde. Sendo assim, a utilização de produtos naturais deve ser sistematicamente investigada pelos profissionais de saúde que lidam com doentes medicados com varfarina (nível de evidência C). ${ }^{19}$

No que se refere às interacções da varfarina com produtos naturais, existem alguns estudos que demonstraram interacções in vitro. ${ }^{20,21}$ No entanto, a importância destes achados para a prática clínica (interacções in vivo) ainda é uma incógnita. Isto é, pelo facto duma substância activa contida numa planta ou num medicamento natural ter sido capaz de interagir com o citocromo $\mathrm{P}_{450}$ CYP2C9 in vitro, não significa que consiga atingir concentrações suficientes, a nível hepático, para interferir com este citocromo in vivo. ${ }^{5}$

Até à data, a evidência disponível em relação às interacções com os produtos naturais consiste, quase na totalidade, em relatos de casos. Desta forma não constituem uma evidência válida e fidedigna que nos permita determinar uma relação de causa-efeito. ${ }^{5}$
O único produto natural para o qual existe evidência comprovada de interacção com a varfarina é a erva de São João (chá de hipericão). ${ }^{5,22}$ Um ensaio clínico aleatorizado controlado ${ }^{22}$ mostrou que a erva de São João é capaz de induzir os citocromos $\mathrm{P}_{450}$ CYP1A2, CYP3A4 e CYP 2C9, aumentando a metabolização da varfarina e inibindo, desta forma, o seu efeito anticoagulante. No entanto, este estudo foi feito em indivíduos saudáveis aos quais foi administrada varfarina e erva de São João, podendo não se adequar à maior parte dos doentes que tomam varfarina (doentes idosos e/ou com comorbilidades e/ou polimedicados).

No que diz respeito aos produtos naturais, para os quais foram relatados casos de possíveis interacções ou se encontrou evidência contraditória (estudos que revelam interacções e outros que as contestam), apresenta-se um quadro (Quadro II), que pretende agrupá-los, para melhor compreensão.

Sendo assim, em relação aos produtos naturais, o único para o qual existe evidência comprovada de interacção com a varfarina é a erva de São João (chá de hipericão). ${ }^{5,22}$ Esta erva, em indivíduos saudáveis, aumenta a metabolização da varfarina e inibe, desta forma, o seu efeito anticoagulante (nível de evidência A). ${ }^{22}$

Resumindo, a varfarina pode interagir com vários alimentos, medicamentos e produtos naturais. Além disso, principalmente no que se refere aos produtos naturais, os estudos ainda não demonstraram a existência de algumas presumidas interacções, de forma consistente. No entanto, também é verdade que ainda não as conseguiram excluir. Sendo assim, alguns autores sugerem que se deve ter cautela ao medicar um doente que toma varfarina. Concretamente, sugerem que, após a introdução de um fármaco novo na medicação habitual do doente, este alerte para esse facto no serviço onde se efectua o controlo da coagulação do sangue, a fim de ser ponderada a necessidade de um controlo mais apertado do INR. Além disso, deve ser dito aos doentes que não tomem nenhum medicamento (incluindo produtos naturais) que não tenha sido prescrito (nível de evidência C). ${ }^{3,7,10}$

\section{CONCLUSÃO}

A varfarina é um anticoagulante oral, utilizado frequentemente, na prática clínica, em doentes com múltiplas comorbilidades e polimedicados. Adicionalmente, tra- 
QUADRO II. Produtos naturais com relatos de casos ou evidência contraditória de interacções com a varfarina $a^{5-7,10,19-22}$

\begin{tabular}{|l|l}
$\begin{array}{c}\text { Antagonismo da } \\
\text { varfarina ( } \downarrow \text { INR) }\end{array}$ & \multicolumn{1}{|c}{$\begin{array}{c}\text { Potenciação da } \\
\text { varfarina ( } \uparrow \text { INR) }\end{array}$} \\
\hline Chá verde & Alho \\
\hline Erva de São João & Coenzima Q10 \\
& Danshen (uma espécie de salva) \\
& Dong quai \\
& Ginkgo biloba \\
& Ginseng \\
& Papain \\
& Vitamina E \\
\hline
\end{tabular}

ta-se de um fármaco com uma janela terapêutica estreita e que exibe uma enorme variabilidade interindividual em termos de dose-resposta.

Este fármaco é alvo de numerosas interacções alimentares e medicamentosas (incluindo produtos naturais) que podem ser responsáveis pelo aumento do seu efeito anticoagulante (que pode cursar com hemorragias graves e/ou fatais) ou pela inibição da sua acção, colocando o doente em risco de sofrer um evento trombótico.

Sendo assim, é tarefa do médico de família conhecer e estar atento às múltiplas interacções da varfarina, de forma a minimizar as consequências adversas das mesmas.

Como foi dito anteriormente, não se encontrou nenhum artigo de revisão baseada na evidência sobre este tema, tão importante para a prática clínica de profissionais de saúde das mais variadas áreas. Os ensaios clínicos aleatorizados controlados e as meta-análises (que enquadram o melhor nível de evidência - nível de evidência A), também são escassos.

Deste modo, são necessários mais estudos que se incluam neste nível de evidência, para conseguirmos tirar conclusões com maior validade científica e que nos permitam orientar melhor a terapêutica com varfarina.

\section{REFERÊNCIAS BIBLIOGRÁFICAS}

1. Ansell J, Hirsh J, Poller L, Bussey H, Jacobson A, Hylek E. The pharmacology and management of the vitamin K antagonists: the Seventh ACCP Conference on Antithrombotic and Thrombolytic Therapy. Chest 2004 Sep; 126 (3 Suppl): 204S-233S.
2. Booth SL, Centurelli MA.Vitamin K: a practical guide to the dietary management of patients on warfarin. Nutr Rev 1999 Sep; 57 (9 Pt 1): 288$-96$.

3. Haemostasis and Thrombosis Task Force of the British Society for Haematology. Guidelines on oral anticoagulation: third edition. $\mathrm{Br} \mathrm{J} \mathrm{Ha}-$ ematol 1998 May; 101 (2): 374-87.

4. Perrero PP, Willoughby DF, Eggert JA, Counts SH. Warfarin therapy in older adults: managing treatment in the primary care setting. J Gerontol Nurs 2004 Jul; 30 (7): 44-54.

5. Greenblatt DJ, von Moltke LL. Interaction of warfarin with drugs, natural substances, and foods. J Clin Pharmacol 2005 Feb; 45 (2): 127-32.

6. du Breuil AL, Umland EM. Outpatient management of anticoagulation therapy. Am Fam Physician 2007 Apr 1; 75 (7): 1031-42.

7. Venkatachalam V, D'Attilio K, Lewis A, Acevedo M. Anticoagulation in ambulatory care: an evidence-based review of the literature. Prof Case Manag 2007 Mar-Apr; 12 (2): 106-11.

8. Ebell MH, Siwek J, Weiss BD, Woolf SH, Susman J, Ewingman B, et al. Strength of recommendation taxonomy (SORT): a patient-centered approach to grading evidence in the medical literature. Am Fam Physician 2004 Feb 1; 69 (3): 548-56.

9. Wittkowsky AK. A systematic review and inventory of supplement effects on warfarin and other anticoagulants. Thromb Res 2005; 117 (12): 81-6.

10. Holbrook AM, Pereira JA, Labiris R, McDonald H, Douketis JD, Crowther $M$, et al. Systematic overview of warfarin and its drug and food interactions. Arch Intern Med 2005 May 23; 165 (10): 1095-106.

11. Institute for Clinical Systems Improvement (ICSI). Antithrombotic therapy supplement. Bloomington (MN): Institute for Clinical Systems Improvement (ICSI); 2007.

12. Franco V, Polanczyk CA, Clausell N, Rohde LE. Role of dietary vitamin $\mathrm{K}$ intake in chronic oral anticoagulation: prospective evidence from observational and randomized protocols. Am J Med 2004 May 15; 116 (10): 651-6.

13. Schaefer MG, Plowman BK, Morreale AP, Egan M. Interaction of rofecoxib and celecoxib with warfarin. Am J Health Syst Pharm 2003 Jul 1; 60 (13): 1319-23.

14. Chaffman MO.Anticoagulation in adult ambulatory patient: basic principles and current concepts in warfarin therapy. Top Geriatr Rehabil 2001 Dec; 17 (2): 18-37.

15. Mahé I, Bertrand N, Drouet L, Bal Dit Sollier C, Simoneau G, Mazoyer $E$, et al. Interaction between paracetamol and warfarin in patients: a double-blind, placebo-controlled, randomized study. Haematologica 2006 Dec; 91 (12): 1621-7.

16. Mahé I, Bertrand N, Drouet L, Simoneau G, Mazoyer E, Bal Dit Sollier $\mathrm{C}$, et al. Paracetamol: a haemorrhagic risk factor in patients on warfarin. Br J Clin Pharmacol 2005 Mar; 59 (3): 371-4.

17. Parra D, Beckey NP, Stevens GR. The effect of acetaminophen on the international normalized ratio in patients stabilized on warfarin therapy. Pharmacotherapy 2007 May; 27 (5): 675-83.

18. Maurer-Spurej E, Pittendreigh C, Solomons K. The influence of selective serotonin reuptake inhibitors on human platelet serotonin. Thromb Haemost 2004 Jan; 91 (1): 119-28.

19. Brazier NC, Levine MA. Drug-herb interaction among commonly used conventional medicines: a compendium for health care professionals. 
Am J Ther 2003 May-Jun; 10 (3): 163-9.

20. Zhou S, Gao Y, JiangW, Huang M, Xu A, Paxton JW. Interactions of herbs with cytochrome P450. Drug Metab Rev 2003 Feb; 35 (1): 35-98.

21. Zou L, Harkey MR, Henderson GL. Effects of herbal components on CDNA-expressed cytochrome P450 enzyme catalytic activity. Life Sci 2002 Aug 16; 71 (13): 1579-89.

22. Jiang $X$, Williams KM, Liauw WS, Ammit AJ, Roufogalis BD, Duke CC, et al. Effect of St John's wort and ginseng on the pharmacokinetics and pharmacodynamics of warfarin in healthy subjects. $\mathrm{Br} J$ Clin Pharmacol 2004 May; 57 (5): 592-9.

\author{
ENDEREÇO PARA CORRESPONDÊNCIA \\ Nuno Lima \\ Centro de Saúde de Paranhos \\ Rua do Vale Formoso, 466 \\ 4200-510 Porto
}

\section{ABSTRACT}

Introduction: Warfarin has been the mainstay of oral anticoagulant therapy. Its effectiveness has been established in various contexts in clinical practice.

Objectives: Review the interactions of warfarin with food, drugs and natural substances/herbs.

Methods: British Medical Journal (BMJ) Clinical Evidence, Evidence Based Medicine online, National Guideline Clearinghouse, National Library of Guidelines, The Cochrane Library, DARE, Bandolier and MEDLINE were searched using the following key words (MeSH terms): warfarin, drug interactions, food interactions and herbal interactions. The search was limited to articles written in English, French, Spanish and Portuguese, published between January 2003 and present.

The Index of Portuguese Medical Journals ("Índex de Revistas Médicas Portuguesas") was also searched using the following key words: "varfarina", "interacções alimentares" and "interacções medicamentosas".

To evaluate the Level of Evidence, the rating system of the American Family Physician (Strength Of Recommendation Taxonomy-SORT) was used: level A (randomized controlled trials/meta-analysis); level B (other evidence) and level C (consensus/expert opinion).

Results and Discussion: Food-Drug Interactions: Foods with a high content of vitamin $\mathrm{K}$ interact with warfarin. Their absolute eviction is not recommended but a stable and moderate ingestion is advised.

Drug Interactions: These are numerous and involve different mechanisms: (1) Reduced warfarin absorption; (2) Potentiation/Inhibition of cytochrome P450 in the liver; (3) Reduction of endogenous vitamin K synthesis by the intestinal flora; (4) Inhibition of platelet aggregation; (5) Increase of the catabolism of vitamin K-dependent coagulation factors; (6) Induction of coagulant factors; (7) Unknown mechanisms.

Herbal drug interactions: St John's wort (hypericum), in healthy subjects, induces warfarin clearance, which in turn inhibits its anticoagulant effect.

Conclusion: Studies with a good level of evidence, concerning an issue of this importance, are scarce. More high quality and long-term controlled and randomized studies about warfarin interactions with foods, drugs and herbs are needed.

Keywords: Warfarin; Drug interactions; Food-drug interactions; Herb-drug interactions. 